\title{
Countering Islamophobia to create a global positive change
}

\author{
Keywords \\ Activism, Awareness, Communication Design, Islamophobia, Social Change.
}

Ignorance and misinformation are often common causes of hatred and discrimination, and change is required to break the cycle so that it may positively influence attitudes and behaviour. Islamophobia is a global trend of hatred towards Islam and Muslims, manifested in social, political and cultural contexts. As a catalyst for positive societal change, communication design can counteract Islamophobia by focusing on creating awareness, educating, and debunking stereotypes by graphically presenting information which invokes empathy and consciousness within the viewer. The methodology of design activism is employed in this research, in which design is used to create a connection between the viewer and a greater cause through graphic language. This practiceoriented research will use the methods of literature analysis, visual representations of concepts and theories, and iterations of design experimentation to create a cohesive awareness campaign all situated in design activism. The aim is to address the thinking, behaviour, actions and consequences behind Islamophobia, in an effort to take a step towards positive global change. Through focusing on changing mindsets, consequential actions have a chance to be altered, creating an opportunity for positive change. Islamophobia is not limited to a certain time or place, therefore the communication design deliverables resulting from this research aim to reach current and future change makers through several platforms. Through digital - a social media campaign, content can reach an extensive range of users and the shareability factor is prominent. And through print - publicly displayed poster design content can be presented to a non-selective public, without the obstruction of tailored algorithms. The content of this campaign will include facts, statistics, stories, and rhetorical questions delivered through typography, information design and layout which aids how effectively the message is communicated. Design is everywhere, connecting with people belonging to several walks of life, therefore outcomes which exist in a range of formats ensures outreach of a wider target audience which is crucial to raising awareness. The use of visual language plays a key role in this research, as a tool of invoking empathy within the viewer and sparking change in a quick and effective manner. This project will also show how change does not have to be monumental or wide-scaled to be important, rather any small difference made within a person, or within their circle, is just as significant. While I recognise that Islamophobia cannot be eradicated overnight, using design to spark awareness and empathy is a small yet significant step in the right direction. 